\title{
Comparative Analysis of the Effectiveness of Some Biological Injected Wound Healing Stimulators and Criteria for Its Evaluation
}

This article was published in the following Dove Press journal:

Drug Design, Development and Therapy

\author{
Ekaterina Vladimirovna Silina (D) \\ Natalia Evgenievna Manturova (iD) ${ }^{2}$ \\ Petr Frantsevich Litvitskiy ${ }^{3}$ \\ Victor Aleksandrovich Stupin (iD) ${ }^{4}$ \\ 'Department of Human Pathology, I.M. \\ Sechenov First Moscow State Medical \\ University (Sechenov University), \\ Moscow I 1999I, Russia; ${ }^{2}$ Department of \\ Plastic and Reconstructive Surgery, \\ Cosmetology and Cell Technologies, \\ Pirogov Russian National Research \\ Medical University, Moscow II7997, \\ Russia; ${ }^{3}$ Department of Pathophysiology, \\ I.M. Sechenov First Moscow State \\ Medical University (Sechenov University), \\ Moscow I I999I, Russia; ${ }^{4}$ Department of \\ Hospital Surgery №l, Pirogov Russian \\ National Research Medical University, \\ Moscow II7997, Russia
}

Purpose: To investigate the comparative effectiveness of certain biological injectable stimulants for the healing of skin wounds and criteria for its assessment.

Materials and Methods: A comparative study of the effectiveness of mesenchymal stem cells (SC group), collagen (Collagen group), and deproteinized calf blood hemoderivative (DCBH group) was carried out using an acute wound model. Control wounds were injected with isotonic sodium chloride solution (Control group). A total of four groups (28 wounds per group) were included in the study. Aged male Wistar rats were used as experimental animals. A dynamic assessment of the wound areas and edges, microvasculature assessment via laser Doppler flowmetry, histological and morphometric analyses to determine the quantitative and qualitative fibroblasts composition, as well as the degree of newly synthesized collagen maturity, was conducted on days $0,3,7$, and 14 .

Results: The administration of SCs provided a rapid but short-lasting effect, whereas the administration of collagen resulted in a delayed but long-lasting wound-healing effect. DCBH resulted in little to no effect. An increase in the perfusion volume of the wound edges accelerated the regeneration process, while the level of microcirculation did not affect the number and activity of fibroblasts. The wound healing acceleration, as well as the new collagen and stratified epithelium formation and maturation, was associated with the presence of a sufficient pool of mature and active fibroblasts in the wound, and not with the number of fibroblasts.

Conclusion: The present results clarify the action mechanisms of the studied drugs. In addition, the application purposes and different effects of each drug on the different wound healing phases were demonstrated. An assumption on the multi-component treatment advisability under the wound condition objective assessment possibility was made. Findings from this study may assist clinicians in making an informed transition to personalized wound management and achieve better clinical outcomes.

Keywords: collagen, deproteinized calf blood hemoderivative, mesenchymal stem cell, fibroblast, microhemocirculation, wound

\section{Introduction}

Acute and chronic wound treatment will always remain an urgent task. The increasing number of drugs and methods for the treatment of wounds confirms this fact and indicates the dissatisfaction of clinicians with the final results. ${ }^{1-5}$ Hence, fundamental study of the drug action mechanisms and the regeneration processes stimulating wound healing, alongside the development of personalized medicine, will contribute to the targeted and consequently more effective and safe use of drugs.
Correspondence: Ekaterina Vladimirovna Silina Department of Human Pathology, I.M. Sechenov First Moscow State Medical University (Sechenov University),

Trubetskaya Str, 8, Moscow I1999I,

Russia

Email silinaekaterina@mail.ru
Drug Design, Development and Therapy 2020:14 4869-4883 
It is established that angiogenesis is an important repairing mechanism. ${ }^{6-9}$ The new microcapillary network growth provides nutrition for the new tissues and creates an environment for the normal cell life that restores damaged organ functions. Angiogenesis regulates the transition from the inflammation phase to the healing wound extracellular framework formation, including fibroblasts functioning. ${ }^{7-10}$ The presence of fibroblasts in the wound, as well as their multiplication and maturation to the stage of a mature cell (which has a rich cytoplasmic apparatus) is necessary for the intercellular proteins, including type I collagen synthesis. ${ }^{11-14}$ In turn, the population of dermal fibroblasts is involved in the regulation of cell proliferation, including endothelial cell proliferation, through direct intercellular or autocrine/paracrine interactions. ${ }^{8,14}$ Thus, for an objective assessment of the effectiveness of the drug, it is necessary to use these two most accurate morphological markers. These biomarkers, which reflect wound overgrowth and nutrition of its tissues, allow physicians to draw conclusions on wound repair and predict scar formation after its closure. However, in clinical practice, in the wound healing process, dynamic quantitative and qualitative assessments of newly formed vessels, fibroblast cells, and wound collagenization are not carried out directly (histological studies). Therefore, experimental studies on animals continue to be relevant.

To address these issues, a pilot study aimed at investigating the comparative effectiveness of certain injectable stimulants of biological origin for the healing of skin wounds and criteria for its assessment. The acute cutaneous wound model is the prototype for numerous small interventions performed under local anesthesia in cosmetology and surgery. Various injectable drugs of different action mechanisms were chosen to be injected into the wound during surgical treatment. All selected injectables were of biological origin and derived from mammals; their effectiveness in wound treatment has been previously proven in clinical and experimental studies. These include collagen $\left(\right.$ Collost $^{\circledR}$; which contains native unreconstructed collagen obtained from the skin of cattle), ${ }^{15-19}$ deproteinized calf blood hemoderivative (DCBH; Actovegin $\left.{ }^{\circledR}\right),{ }^{20-22}$ and mesenchymal stem cells (SCs). ${ }^{23-28}$ An obvious advantage of the injectable drugs is the ability to accurately administer a certain volume and dose of the drug to the same location in all animals, providing identical wound treatment conditions. Since the problem of wound healing is most acute in adults and the elderly, the experiment was carried out using adult aging rats.

\section{Materials and Methods}

The experiment was conducted on male Wistar rats, aged 9 months, weighing in averaged (median [Me]): $433 \mathrm{~g}$ (Q1/ Q3: 390/475 g). Square wounds were modeled on the backs of the rats using a patented device that allows the creation of cutaneous wounds of standardized size in experimental animals (Patent RF No. 79,701/10.01.2009). The operation was performed under general anesthesia (intraperitoneal chloral hydrate: $300 \mathrm{mg} / \mathrm{kg}$ ) under nonsterile conditions. A total of 112 wounds were modeled. The wound area (10 min after modeling) in all rats averaged $123 \mathrm{~mm}^{2}$ (Q1/Q3: $116 / 140 \mathrm{~mm}^{2}$ ), while the wound depth reached the latissimus dorsi muscle fascia.

Prior to the experiment, the rats were maintained in quarantine for 2 weeks under standard vivarium conditions with a 12/12 light/dark cycle and free access to water and food. Immediately after randomization (1 day prior to wound modeling), the animals were isolated (one per cage) to exclude the influence on wounds from other rats.

Study checkpoints were on day 0 (wound modeling, measurements, drug or control solution injections) and days 3, 7, 14 (wound healing dynamic assessment). The animals were withdrawn from the experiment on days 3, 7, and 14 under general anesthesia (intraperitoneally chloral hydrate: $300 \mathrm{mg} / \mathrm{kg}$ ). Rapid exsanguination was performed by opening the right ventricle of the heart.

\section{Study Groups and Animal Labeling}

Age- and sex-matched rats (9-month-old males) were randomized by weight 1 day prior to the experiment. Each rat was assigned a group number and withdrawal day. Therefore, initially, all groups were comparable in terms of weight and withdrawal time. For each control day, seven wounds were marked in each group. The group and time labelings were performed on the tail of the animal prior to the experiment.

The results of the four wound treatment groups (28 wounds in each group) were split according to the specifics of the injected drug. The volumes and injection techniques were identical for all wounds. On day 0 , the same volume of drugs $(0.2 \mathrm{~mL})$ was injected into the wounds of all groups. It was of fundamental importance, because injecting more of the drug could potentially increase compression of the surrounding tissue and slow down the healing process. Through a single puncture in the lower 
outer corner of the wound edge, drug used in clinical practice was injected into the lateral $(0.1 \mathrm{~mL})$ and caudal edges of the wound $(0.1 \mathrm{~mL})$. In the Control group, isotonic $0.9 \%$ sodium chloride solution was injected into the wound edges on the modeling day. In the SC group, a culture of mesenchymal SCs isolated from the human umbilical cord at a concentration of $0.5 \times 10^{6}$ per $\mathrm{mL}$ (100,000 SCs per wound) was injected into the wound edges on the modeling day. In the Collagen group, collagen (Collost ${ }^{\circledR}$ solution containing 7\% collagen gel from cattle skin) was injected into the wound edges on the modeling day. In the DCBH group, DCBH (Actovegin ${ }^{\circledR}$ medicine at a concentration of $40 \mathrm{mg} / \mathrm{mL}$ ) was injected into the wound edges on the modeling day.

\section{Stem Cell Production Technology}

To obtain stem cells, a section of the human umbilical cord was used as the primary biological material, which was obtained after timely delivery without complications and when assessing the newborn on the Apgar scale of at least 8 points. Prerequisite was to obtain informed consent from the prospective mother. Some requirements were imposed on the woman in labor: the pregnancy had to proceed without complications, the woman in labor did not have serious somatic diseases and was not a carrier of dangerous viral or bacterial infections. Before giving birth, the donor was tested (for hepatitis viruses, human immunodeficiency viruses, herpes types 1, 2 and 6, Epstein-Barr, Cytomegalovirus, Toxoplasma Gondi, syphilis).

In the first 10 minutes from the moment of bandaging and cutting the umbilical cord, a fragment of the umbilical cord was taken. The umbilical cord section had to be at least $10 \mathrm{~cm}$ in length and contain at least $10 \mathrm{~mL}$ of whole blood (for subsequent testing). It was placed in a container filled with Hanks solution with added antibacterial agents. Transportation of the biomaterial to the cell laboratory was carried out in the first 4 hours after delivery in this container filled with melting ice, so the temperature of the material did not rise above $+8^{\circ} \mathrm{C}$, and did not fall below $+2^{\circ} \mathrm{C}$. Direct isolation and cultivation of cell culture was performed according to a previously developed technique that was used in other studies. ${ }^{28-30}$ This made it possible to obtain cellular material of a standard morphological structure and further compare the results of different studies with each other. The cells were grown in a-MEM culture medium (Lonza), StemPro тм MSC (Gibco, USA) to a concentration of 500,000 cells per $1 \mathrm{~mL}$. The number of passages: 4. Cell culture typing was performed by microscopy of unstained preparations and by detection of specific protein markers of cytophenotypes by immunocytochemistry and flow cytofluorometry. Each new cell culture was tested for the presence of histocompatibility antigens (HLA-ABC, HLA-DR), as well as surface marker proteins of mesenchymal stem cells: CD34, CD44 (HCAM), CD45, CD49b ( $\alpha 2 \beta 1$-integrin), CD54 (ICAM), CD90 (Thy-1), CD105 (endoglin), CD106, CD117 (c-kit). Despite the fact that the mother was tested for virus and bacterial carriage, it was considered necessary to retest the resulting culture of umbilical cord cells (virological and bacteriological testing) for the presence of microflora, markers of hepatitis $\mathrm{B}$ and $\mathrm{C}$ viruses, HIV, herpes simplex virus types 1, 2 and 6, Epstein-Barr, cytomegalovirus and toxoplasmosis.

\section{Research Methods}

Different indices were dynamically analyzed, including the changes in wound areas (on days $0,1,3,7$, and 14), level of microhemocirculation in the wound edges (in anesthetized rats on days $0,3,7$, and 14; twice in each animal, on the modeling and withdrawal days). Moreover, staining of wound skin histological sections was performed using different methods followed by light microscopy, as well as morphometry with the resident fibroblast cells count during animal withdrawal (on days 3, 7, and 14).

The wound size measurement technique consisted of several stages. Firstly, visual examination, linear wound measurement, and capture of images with EOS550D camera (Canon, Japan) were performed at the same angle and distance of $30 \mathrm{~cm}$ to the wound with the same ruler used at all points of the experiment. Subsequently, the wound area index was calculated, clearly limited by its edges, expressing the final result in $\mathrm{mm}^{2}$. For this, we used the JMicroVision 1.2.7 program (Switzerland).

According to the measurements (Me and interquartile range $[\mathrm{IQR}]$ ), the wound area on the modeling day did not statistically differ between the groups (Control group, Me: $128 \mathrm{~mm}^{2}$ [IQR: 106-137 $\mathrm{mm}^{2}$ ]; SC group, Me: $134 \mathrm{~mm}^{2}$ [IQR: 122-155 $\mathrm{mm}^{2}$ ]; Collagen group, Me: $130 \mathrm{~mm}^{2}$ [IQR: 119-144 $\mathrm{mm}^{2}$ ]; and DCBH group, Me: $126 \mathrm{~mm}^{2}$ [IQR: $110-131 \mathrm{~mm}^{2}$ ]; $\mathrm{p}>0.05$ ).

Microhemocirculation was analyzed in anesthetized rats using the MP150 hardware and software complex for electrophysiological studies (BIOPAC Systems, Inc, USA). The complex included an LDF100C laser Doppler flowmetry module equipped with a TSD145 needle sensor and AcqKnowledgeversion 4.4.1 software (Figure 1). It 


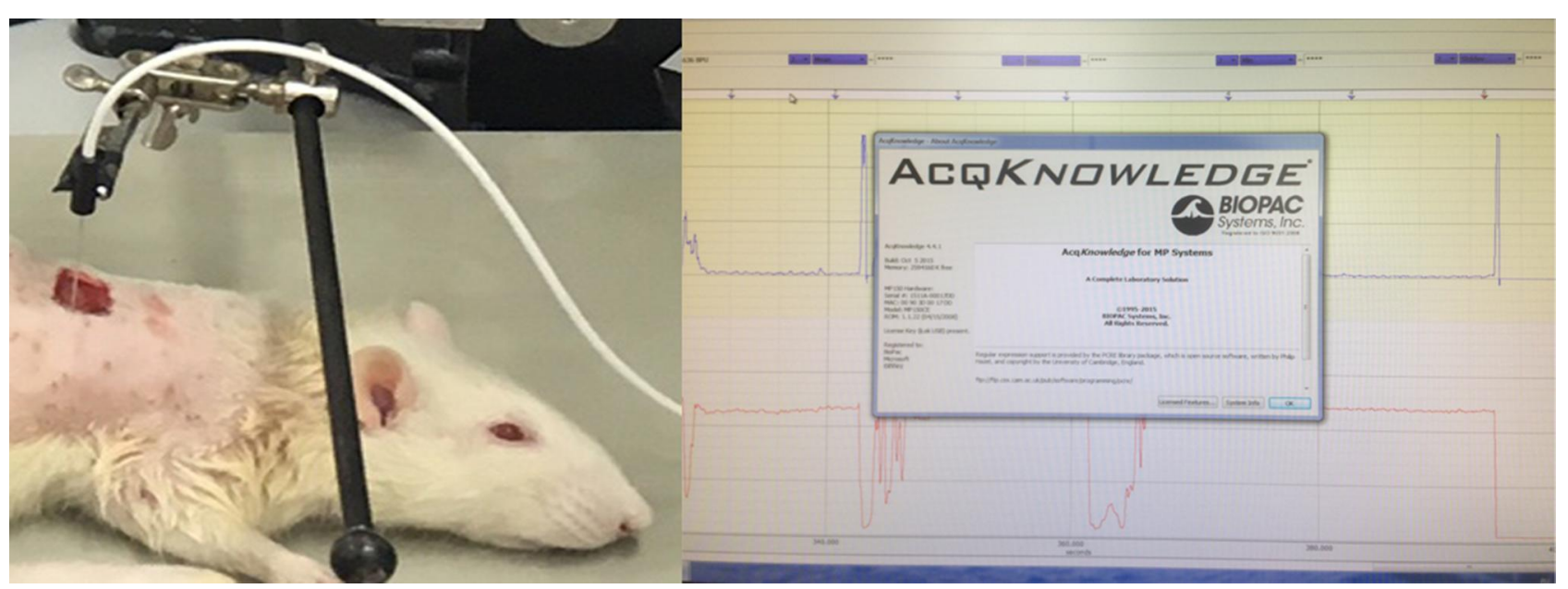

Figure I Microhemocirculation analysis of the edges of a wound in a rat under general anesthesia.

detects the presence of reflected signal frequency shifts and, using the software signal processing algorithm, converts them into perfusion units (ie, blood perfusion units [BPU]). The needle probe was sequentially installed perpendicularly to the skin $2 \mathrm{~mm}$ outwards along all four edges of the wound. The final result was the arithmetic mean of the BPU obtained from all edges of the wound.

This article presents a comparative analysis of the microhemoperfusion volume dynamics in animals withdrawn from the experiment at the control points in comparison with the initial volume measured on day 0 . The microhemoperfusion values were statistically indistinguishable in the study groups on day 0 .

For histological analysis, three consecutive dissections of each wound were made. On each dissection were identified the wound center and edges. Sections were stained differently: hematoxylin-eosin (for assessment of wound epithelialization under descriptive light microscopy); hematoxylin (processing using the Image-J program [National Institutes of Health, Bethesda, MD, USA] for the quantitative analysis of fibroblast cells according to their morphological characteristics in granulation layers); and Van Gieson (to assess the wound collagenization, including the collagen location, volume, and degree of maturity). Microscopy was performed at $\times 40, \times 100$, and $\times 400$ magnifications using a Leica CME microscope (Germany) and an eyepiece camera.

\section{Ethical Considerations}

The experiment was performed according to the principles of laboratory animals handling and complied with the provisions of the "European Convention for the
Protection of Vertebral Animals Used for Experimental and Other Scientific Purposes. CETS 123". The study was approved by the Regional Ethics Committee of the FSBEI of HE Kursk State Medical University under the Ministry of Health of the Russian Federation (Protocol No. 5 dated 02.11.17). Mesenchymal SCs used in the experiment were isolated from a human umbilical cord after a normal birth at 38-40 weeks of the gestation period with the informed consent of the donors.

\section{Statistics}

Statistical analysis was performed with the SPSS version 23.0 software (IBM Corp., Armonk, NY, USA) using standard parametric and non-parametric criteria. Continuous quantitative data were descriptively presented as the median (Me), as well as the lower $25 \%$ and upper $75 \%$ quartiles. Qualitative data were presented in the form of a percentage. The normality of the quantitative samples distribution was assessed using the Kolmogorov test. The Kruskal-Wallis test was used to compare more than two independent nonparametric samples, followed by an appropriate post hoc analysis (Dunnett's $T$-test for comparison with a control group, for other pairwise comparisons, the Bonferroni test was used). Since a p-value between 0.05 and 0.1 can be considered a trend, we performed a posteriori analysis after obtaining the Kruskal-Wallis test result $0.05-0.1$. This was done to eliminate the possibility of a Type I error. The Wilcoxon test was used for the analysis of two dependent samples. Qualitative characteristics were compared according to the $\chi^{2}$ criterion (analysis of contingency tables). A $\mathrm{p}<0.05$ denoted statistically significant differences. 


\section{Results}

\section{State of the Wounds by the End of Day 3}

By the end of day 3, the wound area of the Control group was the largest (mean: $145 \mathrm{~mm}^{2}$ [IQR: $128-158 \mathrm{~mm}^{2}$ ]), which is on average $1.14 \times$ times more than on day $0(10$ min after wound modeling). However, a significant difference from the Control group wounds was observed only in the $\mathrm{SC}$ group (on average, $1.18 \times$ times less; $\mathrm{p}=0.001$ ). The Me wound area of the SC group was $122 \mathrm{~mm}^{2}$ (IQR: $107-134 \mathrm{~mm}^{2}$ ). The SC group was the only group in which, on day 3 , the wound area decreased significantly compared with that noted on the modeling day. The wound areas of the Collagen and DCBH groups did not statistically differ from the Control group on day 3 , although they were smaller on average (Collagen group, Me: $137 \mathrm{~mm}^{2}$ [IQR: 124-150 mm ${ }^{2}$ ]; DCBH group, Me: $131 \mathrm{~mm}^{2}$ [IQR: $\left.110-146 \mathrm{~mm}^{2}\right] ; \mathrm{p}>0.05$ ). In addition, the wound areas in these groups did not differ from each other and their sizes measured on the modeling day, insignificantly exceeding the size by merely $5-10 \mathrm{~mm}^{2}$. Comparison of all groups did not reveal statistical differences according to the Kruskal-Wallis test $(\mathrm{p}=0.075)$, post hoc tests revealed a difference between the Control and SC groups $(\mathrm{p}=0.002$; the appropriate Dunnett's post hoc test) (Figure 2).

On day 3 , the blood perfusion volume of microvessels in the skin around the wound did not demonstrate significant changes compared with the volumes observed on day 0 in the Control, Collagen, and SC groups ( $p>0.05)$. At the same time, in $43 \%$ of the wounds in the SC group, the perfusion volume tended to increase, whereas it did not

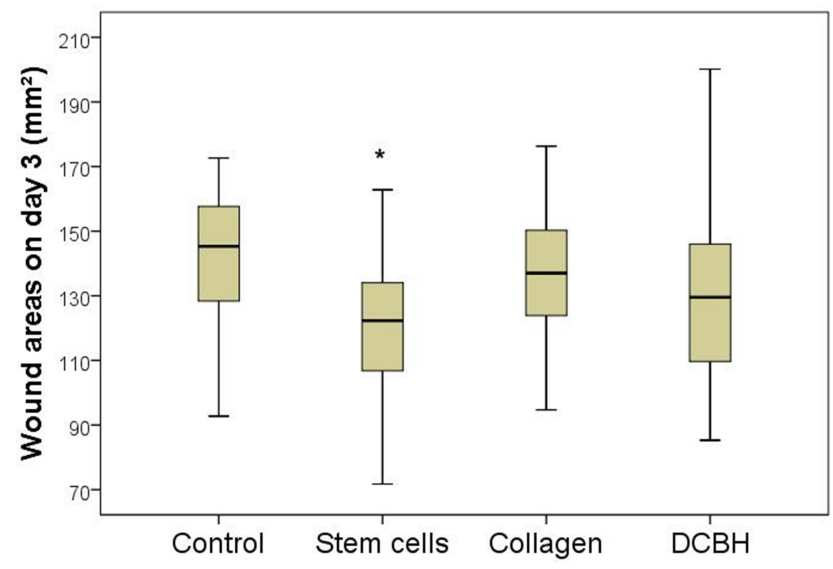

Figure 2 Wound areas on day 3 of the study in different groups $\left(\mathrm{mm}^{2}\right)$. Note: *Difference from the Control group at $p<0.05$ (the appropriate Dunnett's post hoc test).

Abbreviation: $\mathrm{DCBH}$, deproteinized calf blood hemoderivative. change or decreased insignificantly in the remaining $57 \%$ of wounds. A statistically significant increase in the microcirculation volume by $25 \%$ was recorded only in the wound tissues of the DCBH group (on average from 110 BPU on day 0 to 138 BPU on day $3 ; \mathrm{p}<0.05$ ). This indicator increased in $86 \%$ of wounds ( 6 of 7 animals were withdrawn from the experiment on day 3 ). In the other groups, the microcirculation volume did not statistically differ and ranged 115-121 BPU (Figure 3).

It was hypothesized that the change in the microcirculation level under the use of different drugs is associated with different mechanisms of damaged tissue restoration. This hypothesis was confirmed by morphometric data.

During histological wound sections analysis on day 3, the leukocyte numbers in damaged tissues significantly exceeded the concentration of fibroblasts, indicating the exudative inflammation process. According to the number of fibroblasts in all wound parts on day 3, the groups can be arranged in the following sequence (in descending order): Control $>$ DCBH $>$ Collagen $>$ SC. Young fibroblast forms were determined morphometrically on day 3 . The reverse group sequence was observed during the fibroblast concentration ratio analysis in the wound center and edges. In the Control group, the numbers of fibroblasts in the wound center and edges were similar (on average, 117 and 107 fibroblasts per $\mathrm{mm}^{2}$ of wound, respectively). In the DCBH group, the number of fibroblasts in the wound center was $1.5 \times$ times higher than that recorded in the periphery (on average 111 and 76 fibroblasts per

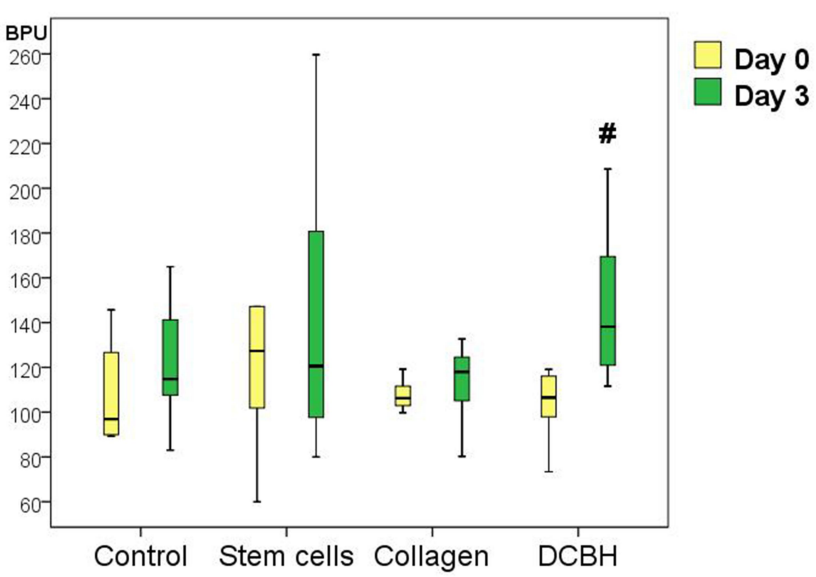

Figure 3 Microhemocirculation volume in the edge of wounds on days 0 and 3 in different groups (BPU).

Note: $\# p<0.05$, significant difference in the dynamics of the associated indicator within a separate group relative to day 0 (Wilcoxon test).

Abbreviations: BPU, blood perfusion units; $\mathrm{DCBH}$, deproteinized calf blood hemoderivative. 
Table I The Number of Fibroblasts in the Center and Edge of the Wounds in Different Groups on Day 3 (n)

\begin{tabular}{|l|l|l|}
\hline Group & Center & Edge \\
\hline Control & $117[108-121]$ & $107[92-109]$ \\
Stem cells & $98[92-117]$ & $18[14-49] * \#$ \\
Collagen & $101[95-106]$ & $52[39-59]^{*}$ \\
Deproteinized calf blood hemoderivative & $111[101-118]$ & $76[74-97] \#$ \\
$\mathrm{P}$ (Kruskal-Wallis test) & 0.168 & 0.003 \\
\hline
\end{tabular}

Notes: Data are presented as the median and interquartile range [lower $25 \%$-upper $75 \%$ quartile]. *Difference from the Control group at $p<0.05$, Dunnett's test; \#p<0.05, significant difference between SC and DCBH groups, the appropriate post hoc Bonferroni test.

$\mathrm{mm}^{2}$ of wound, respectively). In the Collagen group, the difference in fibroblast concentration averaged $1.9 \times(\mathrm{Me}$ : 101 and 52 cells $/ \mathrm{mm}^{2}$, respectively). At the bottom of the wound areas in the SC group, the number of fibroblasts was $5.4 \times$ times higher than that measured at the edges (Table 1).

Histological sections stained according to Van Gieson revealed that, on day 3 in all wounds under a formed thick scab, fibrin filled the wound crater; the fibrin contained few thin, young, and weakly stained collagen fibers. With the production of a weak protein by fibroblasts on day 3 , the least amount of immature young collagen was detected in wounds of the DCBH group. Newly formed collagen was observed in the SC and Collagen groups. According to wound collagenization, groups can be arranged in the following order (from minimum to maximum): $\mathrm{DCBH}<$ Control $<\mathrm{SC}<$ Collagen (Figure 4).

Generally, in the Collagen group, by the end of day 3, better formation of granulation tissue and layered organization were observed compared with the other groups. Moreover, in half of the Collagen group cases, a structured collagen band running from the edges to the center of the
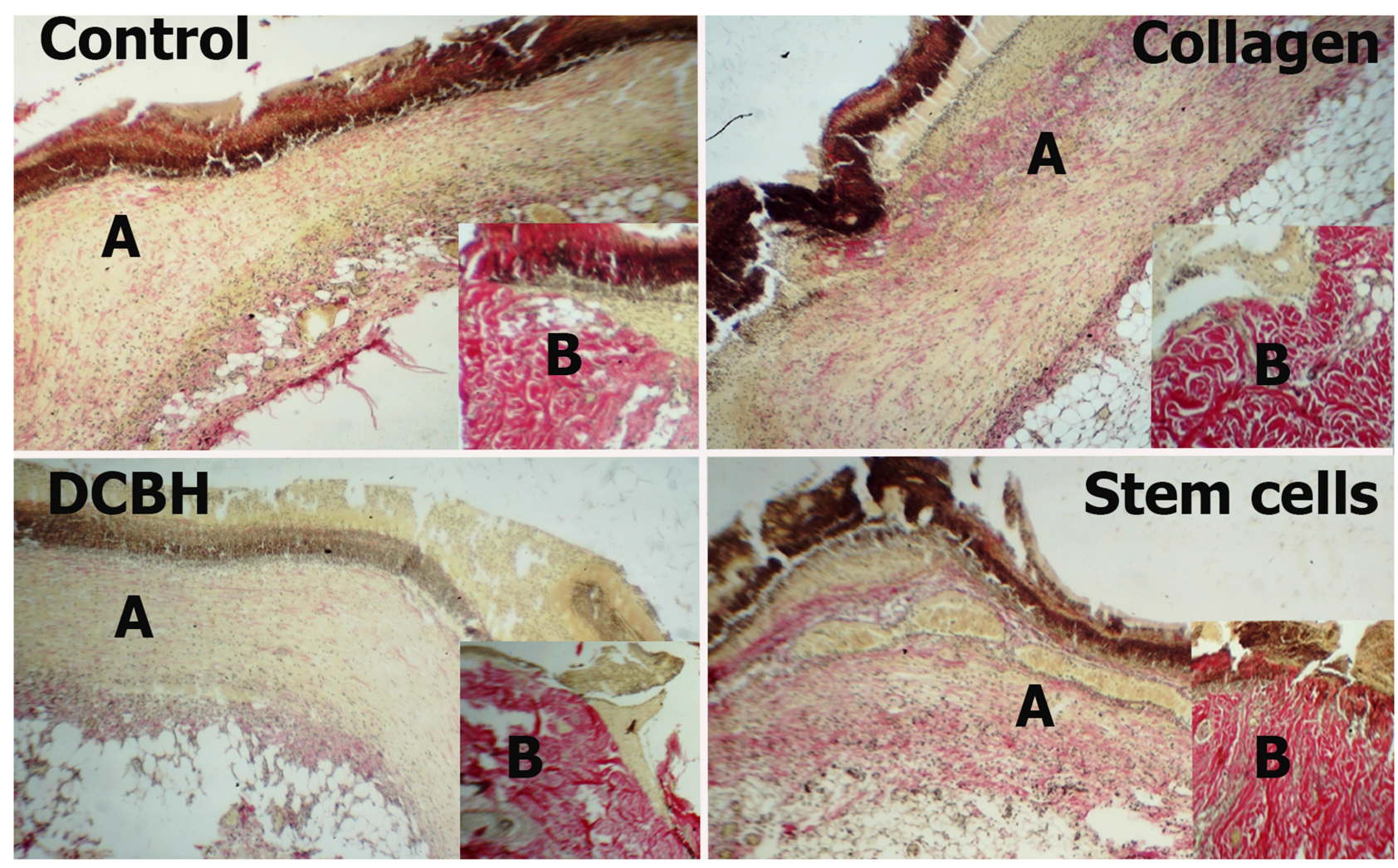

Figure 4 Wound areas of different groups on day 3. Light microscopy, Van Gieson staining, magnification: $\times 40$.

Notes: "A" Indicates the wound center impregnated with fibrin and a small number of thin pale unstructured collagen fibers. "B" Indicates an intact wound edge with brightcolored structured dense mature collagen.

Abbreviation: DCBH, deproteinized calf blood hemoderivative. 
wound was detected, probably due to the drug injected into the wound. Although the band was brighter than those observed in other wound sites, it differed significantly in color and thickness from intact collagen in the same wound edges. This may be due to the activity of amino acids of the introduced foreign collagen for synthesis.

\section{State of the Wounds by the End of Day 7}

By the end of day 7, the wound areas diminished compared with those measured on the modeling day. Visually, wounds in the Collagen group healed better, whereas those of the Control group exhibited worse healing (Figure 5). Thus, the wound areas of the Collagen group decreased by $1.5 \times$ on average and averaged $85 \mathrm{~mm}^{2}$ (IQR: $72-96 \mathrm{~mm}^{2}$ ). On day 7, the wound areas of the Control and SC groups were statistically significantly larger than those recorded on the modeling day $\left(1.35 \times\right.$; Me: $115 \mathrm{~mm}^{2}$ [IQR: $\left.97-125 \mathrm{~mm}^{2}\right] ; \mathrm{p}=0.003$ ). The DCBH and SC groups did not statistically differ versus the other groups on day 7 of the experiment. Comparison of all groups did not reveal statistical differences according to the Kruskal-Wallis test $(p=0.055)$, the appropriate post hoc tests revealed a difference between the Control and Collagen groups $(\mathrm{p}=0.025)$.

By the end of day 7, the level of microcirculation in the $\mathrm{SC}$ group tissue increased relative to the modeling day by $34 \%$ on average (from $117 \mathrm{BPU}$ on day 0 to $158 \mathrm{BPU}$ on day $7 ; \mathrm{p}<0.05)$. The level of microcirculation in the Collagen group tissue increased to the maximum extent by $43 \%$ on average (from $124 \mathrm{BPU}$ on day 0 to $178 \mathrm{BPU}$ on day $7 ; \mathrm{p}<0.05)$. In the Control and DCBH groups, the tissue perfusion volume of the wound edges did not differ

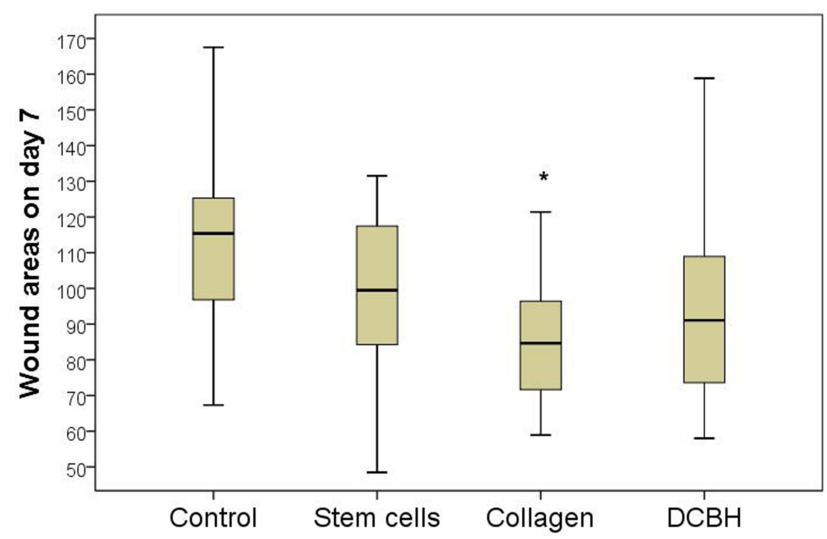

Figure 5 Wound areas on day 7 in different groups $\left(\mathrm{mm}^{2}\right)$.

Note: *Difference from the Control group at $p<0.05$ (the appropriate Dunnett's post hoc test).

Abbreviation: $\mathrm{DCBH}$, deproteinized calf blood hemoderivative. from the initial level, and averaged 133 BPU and 125 BPU, respectively $(p>0.05)$. Notably, in the DCBH group, the tissue microcirculation level around the wound on day 3 was $1.11 \times$ higher than that reported on day 7 , it decreased to its initial level (Figure 6).

The increased blood perfusion in wound edge tissue on day 7 correlated with the smallest wound area, as observed in the SC and Collagen groups. Consequently, an increase in the tissue blood perfusion volume of the wound edge accelerated regeneration, and may serve as a marker of positive outcome.

In the DCHB group, the early recorded positive perfusion effect had ended by the end of day 7 , and was equal to that noted in the control animals, without differing from the microcirculation level established on day 0 .

On day 7, changes in the wound tissues were directly associated with an increase in fibroblast density, which was mainly expressed at the wound edges. The number of fibroblasts at the wound edges increased in all animal groups by $5-33 \times$, compared with those recorded on day 3 (mean: $5.0 \times, 7.2 \times, 9.9 \times$, and $33 \times$ in the Control, DCBH, Collagen, and SC groups, respectively; $\mathrm{p}<0.01$ ). Therefore, on day 7 (versus day 3), fibroblasts prevailed over the other cells, providing the resources for postoperative matrix formation and wound collagenization. Thus, on day 7 , the proliferative regeneration process changed the exudative inflammation period, which was presented on day 3 of the wound healing.

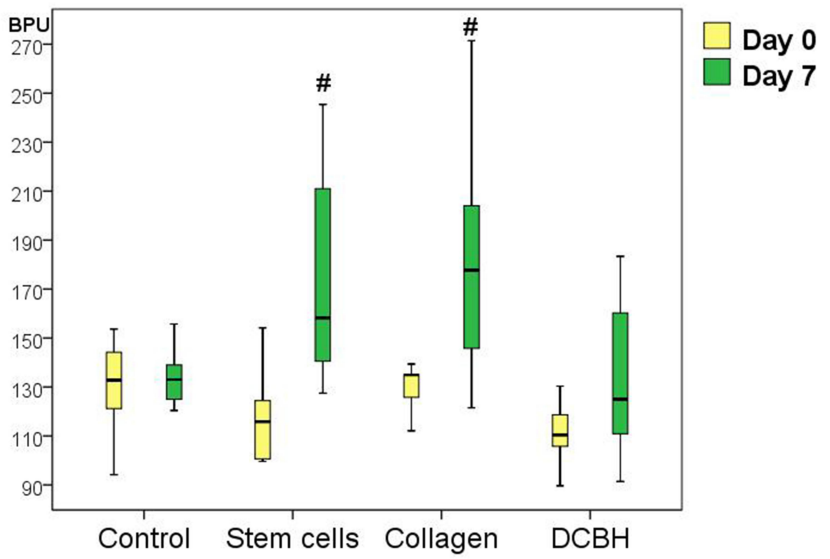

Figure 6 The level of microhemocirculation in the tissue of wound edges on days 0 and 7 in different groups, from animals withdrawn from the experiment on day 7 (BPU).

Note: $\# p<0.05$, significant difference in the level dynamics within a separate group relative to day 0 (Wilcoxon test).

Abbreviations: BPU, blood perfusion units; $\mathrm{DCBH}$, deproteinized calf blood hemoderivative. 
However, in terms of the absolute number of fibroblasts per $1 \mathrm{~mm}^{2}$ of wound area, significant differences were found between the groups during the analysis of the wound edge tissue preparations. The maximum number of fibroblasts at different maturation stages was observed in the $\mathrm{SC}$ group. On day 7, the average number of fibroblasts was 594 cells per $1 \mathrm{~mm}^{2}$. Therefore, it was $1.11 \times$ $(\mathrm{p}=0.029), 1.16 \times(\mathrm{p}=0.048)$, and $1.09 \times(\mathrm{p}=0.45)$ higher than in the Control, Collagen, and DCBH groups, respectively. All other groups did not differ among themselves in terms of this indicator $(\mathrm{p}>0.05)$ (Table 2).

We emphasize that the fibroblast concentration was maximal at the wound center on day 3. On day 7, the fibroblast concentration was maximal at the wound edges than at the wound bottom in all groups (on average, in the Control group: $1.25 \times, \mathrm{p}<0.05$; SC group: $1.32 \times, \mathrm{p}<0.05$; Collagen group: $1.13 \times, \mathrm{p}>0.05$; and DCHB group: $1.19 \times$, $\mathrm{p}<0.05)$. This fact made it possible to trace the initiation of the wound epithelialization process.

On day 7, the marginal epithelialization with epithelial creeping from the periphery to the center was observed in all groups. According to the epidermis organization, the number of layers, and growth bud formation, the SC group demonstrated the best results, followed by the Collagen, Control, and DCBH groups. Of note, the largest difference in the number of fibroblasts between the edges and center of the wound area was observed in the SC group, while the smallest difference was noted in the Collagen group. Only in the Collagen group, there were no significant differences between the number of fibroblasts in different wound sections. An explanation may be that, during treatment with collagen, the regeneration process originated from the wound edges and wound bottom, due to the injected protein, reduced energy, and time required for the formation of the extracellular matrix of the granulation tissue. This accelerated the proliferation stage of the wound healing process.
Additionally, in the SC and Collagen groups, the level of microcirculation in wound edges was the highest, and the total number of fibroblasts was the largest. Moreover, importantly, the cells were the most mature (large process cells with rich organelles and weakly basophilic cytoplasm), and the loose fibrous connective tissue was the most formed and mature. This was mainly observed at the wound edges, promoting the synthesis, formation, and maturation of collagen (Figure 7).

It was found that the ratio of young and mature collagen was equal in $28.6 \%$ of wounds in the $\mathrm{SC}$ and Collagen groups. In the remaining cases, and in $100 \%$ of the wounds in the DCBH and Control groups, young collagen prevailed over mature collagen. The most oxyphilic (brightly colored) was the newly formed collagen in the wound edges of the SC group, followed by the Collagen group. Collagen was least organized in the DCHB and Control groups. This once again confirms the hypothesis that the main mechanism for accelerating wound healing when using collagen is not the stimulation of metabolic processes as such, but the use of the injected collagen as the primary building protein, which is then used by fibroblasts to synthesize their own functioning collagen. A decrease in the wound area by day 3 in the Collagen group occurred due to the long-term non-absorbable collagen injected and an increase in the volume of the wound edges.

\section{State of the Wounds by the End of Day 14}

At 14 days after wound modeling, all wound sizes diminished significantly. Sustaining the 7-day trend, the Collagen group healed better (the wound area decreased $11.8 \times$ in 2 weeks on average), followed by the SC group $(8.9 \times)$ and DCBH group $(8.4 \times)$; the worst result was demonstrated by the Control group (the wound area decreased $4.4 \times$ on average) (Figure 8 ). Thus, the Control

Table 2 The Number of Fibroblasts in the Center and Edge of the Wounds in Different Groups on Day 7 (n)

\begin{tabular}{|l|l|l|}
\hline Group & Center & Edge \\
\hline Control & $428[383-454]$ & $538[488-549]$ \\
Stem cells & $449[395-458]$ & $594[574-638] *$ \\
Collagen & $453[429-479]$ & $514[489-543] \#$ \\
Deproteinized calf blood hemoderivative & $458[442-472]$ & $545[499-561] \#$ \\
P (Kruskal-Wallis test) & 0.425 & 0.021 \\
\hline
\end{tabular}

Notes: Data are presented as the median and interquartile range [lower $25 \%$-upper $75 \%$ quartile]. *Difference from the Control group at $\mathrm{p}<0.05$, Dunnett's test; \#Difference from the SC group at $p<0.05$, the appropriate post hoc Bonferroni test. 


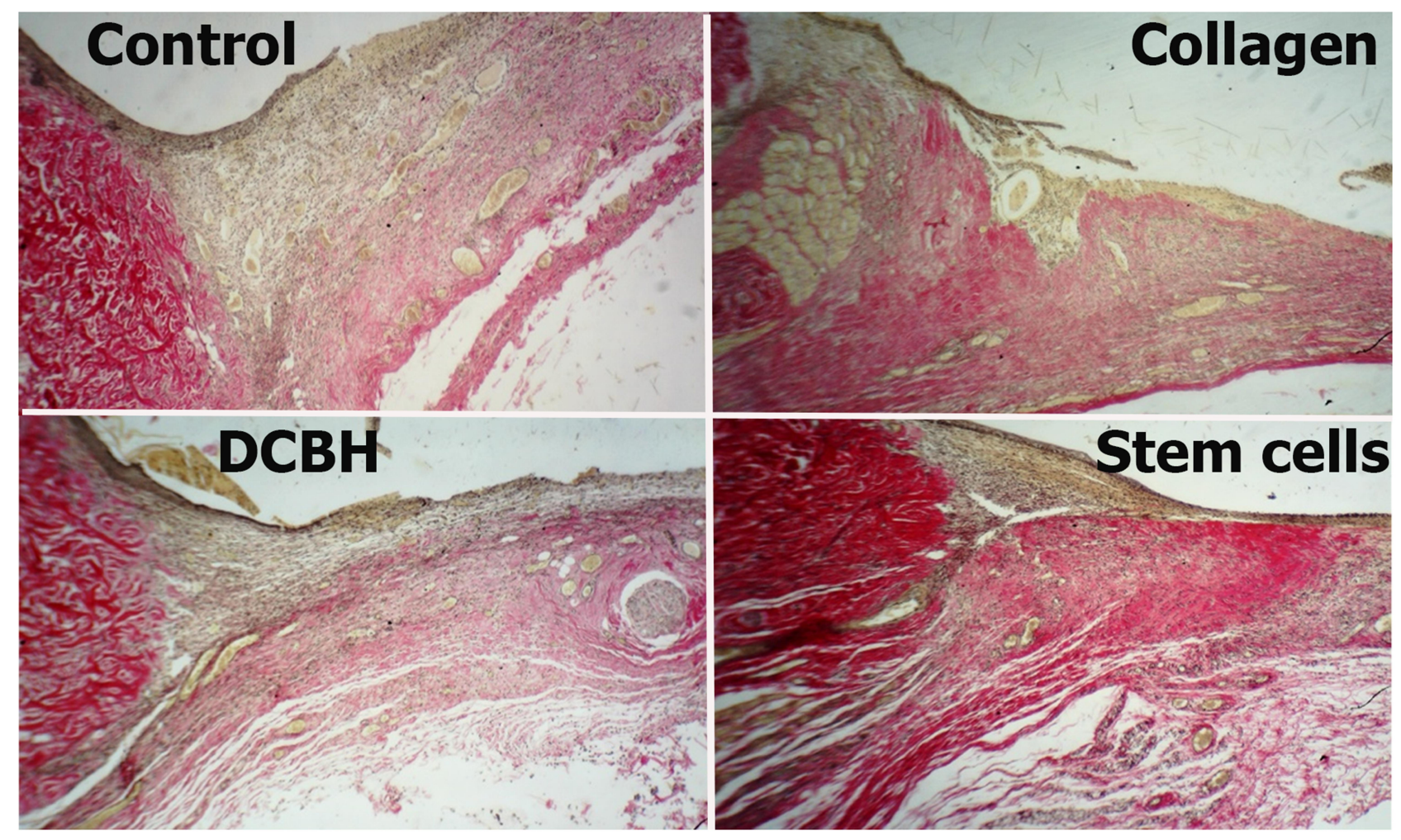

Figure 7 Wound edge tissue in different groups on day 7. Van Gieson staining, magnification: $\times 40$. Abbreviation: DCBH, deproteinized calf blood hemoderivative.

group wound area averaged $26 \mathrm{~mm}^{2}$ (IQR: $18-29 \mathrm{~mm}^{2}$ ). This was higher than in all other pharmacological groups: $1.7 \times$ higher than in the DCBH group (Me: $15 \mathrm{~mm}^{2}$ [IQR: $\left.11-26 \mathrm{~mm}^{2}\right]$ ) and the SC (Me: $15 \mathrm{~mm}^{2}$ [IQR: $\left.10-21 \mathrm{~mm}^{2}\right]$ ), and $2.4 \times$ higher than in the Collagen group (Me: $11 \mathrm{~mm}^{2}$ [IQR: $8-17 \mathrm{~mm}^{2}$ ]). Comparison of

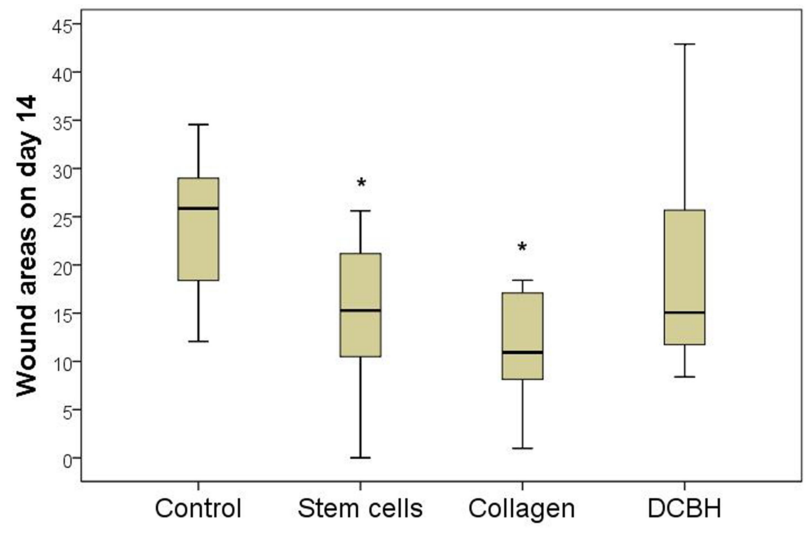

Figure 8 Wound areas on day 14 of different groups $\left(\mathrm{mm}^{2}\right)$.

Note: *Difference from the Control group at $p<0.05$ (the appropriate Dunnett's post hoc test)

Abbreviation: $\mathrm{DCBH}$, deproteinized calf blood hemoderivative. all groups revealed significant differences according to the Kruskal-Wallis test $(p=0.001)$. Dunnett's post hoc test revealed a difference from the Control group: Collagen ( $\mathrm{p}$ $=0.0001)$ and $\mathrm{SC}(\mathrm{p}=0.004)$ groups, no other differences were found in the post hoc tests.

The wound edge tissue perfusion on day 14 increased relative to day 0 in all groups $(\mathrm{p}<0.05)$. The perfusion volume increased to the greatest extent in the Collagen group to $269 \mathrm{BPU}$ (an average of $2.2 \times$ ). This was $1.3 \times$ and $1.6 \times$ higher than in the DCBH group (Me: 208 BPU; $\mathrm{p}<0.05$ ) and SC group (Me: 168 BPU; $\mathrm{p}<0.05$ ), respectively. In the Collagen group, the largest increase in the perfusion volume was revealed by day 14 relative to day 0 . Comparison of all groups on day 14revealed significant differences according to the Kruskal-Wallis test $(\mathrm{p}=$ 0.045). Perfusion volume changed the least in the SC group, by only $1.30 \times$ on average relative to day 0 (Figure 9), and did not change compared with day 7. This may be a consequence of the SC culture action termination during this period of wound healing.

On day 14, the number of fibroblasts under different treatment options prevailed over those of hematopoietic cells. The fibroblast detection frequency at the wound 


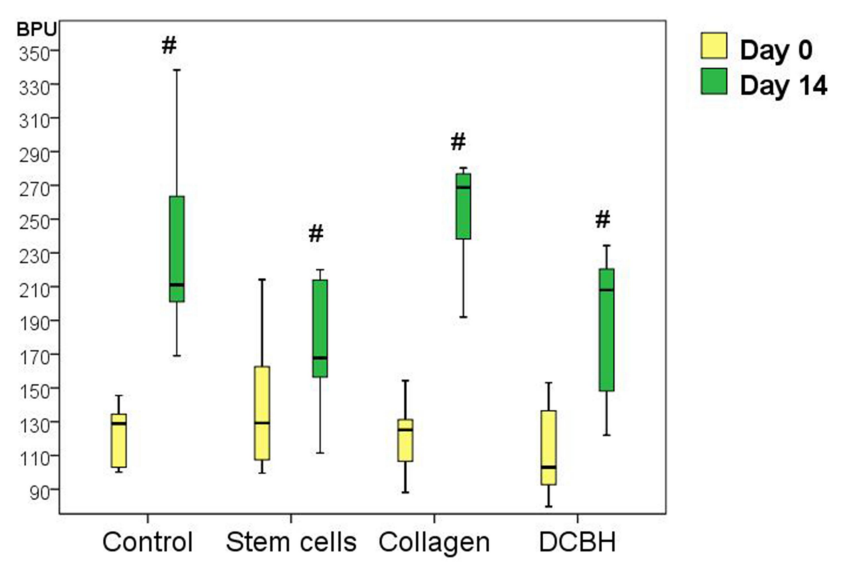

Figure 9 Levels of microcirculation on day 14.

Note: $\# p<0.05$, significant difference in the dynamics of a related indicator within a separate group relative to day 0 (Wilcoxon test).

bottom and edges in all pharmacological groups were higher than that noted in the wound areas of the Control group, which significantly affected the general and morphological profile of the wound healing process. This confirms the conclusion on the effectiveness of regenerating agents for the treatment of wounds in connection with their active involvement in fibroblast secretion and stimulation fibrous loose connective tissue formation, which promotes early wound healing.

The number of fibroblasts was the highest in the wound areas of the Collagen group (center, Me: 595 cells $/ \mathrm{mm}^{2}$; edges, Me: 503 cells $/ \mathrm{mm}^{2}$ ), which were $1.66 \times$ and $1.37 \times$ higher, respectively, than those counted in the Control group $(\mathrm{p}<0.05)$. Of note, the $\mathrm{DCBH}$ group (center, Me: 663 cells $/ \mathrm{mm}^{2}$; edges, Me: 490 cells $/ \mathrm{mm}^{2}$ ) had $1.97 \times$ and $1.34 \times$ more cells, respectively, than the Control group $(p<0.05)$. The lowest concentration of fibroblasts was observed in the SC and Control groups. Only in the Control group, the number of fibroblasts at the edges of the wound area was higher than that in the center (on average, $1.09 \times$ ). In the SC group, the number of fibroblasts was the same in different wound sections. In the other groups, the concentration of fibroblasts was higher in the wound center than in the edges (on average, $1.35 \times$ and $1.18 \times$ higher in the DCBH and Collagen groups, respectively) (Table 3).

The wound epithelialization was evaluated via microscopy of histological sections. The analysis revealed that, by the end of day 14, the best wound healing occurred in the Collagen and SC groups, in which complete epithelization of the entire wound was recorded in $57.1 \%$ and $42.9 \%$ of cases, respectively. The wounds in the Control and DCBH groups did not have complete epithelialization. Marginal epithelialization with a gradual thinning of the epidermis layers did not reach the wound central area. At the same time, in most of the wounds in the Control and DCBH groups, a scab saturated with leukocytes was observed in the wound center. In the Collagen group, the largest number of formed epidermal growth buds was recorded, in which hair follicles with animal hair and sebaceous glands were determined. Under the epidermis, a layer of collagen fibers of varying maturity degrees was detected in all groups. Also, the degree of collagen maturity increased in all groups (compared with the previous control point), especially in the Collagen group (Figure 10).

In the Collagen group, the mature collagen prevailed in numbers over young collagen $(42.9 \%$ of cases). In the remaining $57.1 \%$ of the Collagen group wounds, the young and mature collagen ratio was equal. In other groups on day 14 , there were no cases of mature collagen prevalence in wounds. On day 14, wounds in the Control group showed the least collagenization. In $71.4 \%$ of cases, predominance of young collagen was recorded, and only in $28.6 \%$ an equal ratio of young and mature collagen was recorded. In the SC group, the young collagen predominated in $33.3 \%$ of cases, and an equal ratio of young and mature collagen was observed in $66.7 \%$ of cases. In the DCBH group, $85.7 \%$ of the cases demonstrated an equal ratio of young and mature collagen, while the prevalence

Table 3 The Number of Fibroblasts in the Center and Edge of the Wounds in Different Groups on Day I4 (n)

\begin{tabular}{|l|l|l|}
\hline Group & Center & Edge \\
\hline Control & $337[330-376]$ & $367[322-376]$ \\
Stem cells & $414[399-5 I 4]$ & $408[327-45 I]$ \\
Collagen & $595[498-60 I] *$ & $503[452-638] * \#$ \\
Deproteinized calf blood hemoderivative & $663[579-725] * \#$ & $490[443-519] *$ \\
P (Kruskal-Wallis test) & 0.0001 & 0.002 \\
\hline
\end{tabular}

Notes: Data are presented as the median and interquartile range [lower $25 \%$-upper $75 \%$ quartile]. *Difference from the Control group at $\mathrm{p}<0.05$, Dunnett's test; \#Difference from the SC group at $\mathrm{p}<0.05$, the appropriate post hoc Bonferroni test. 

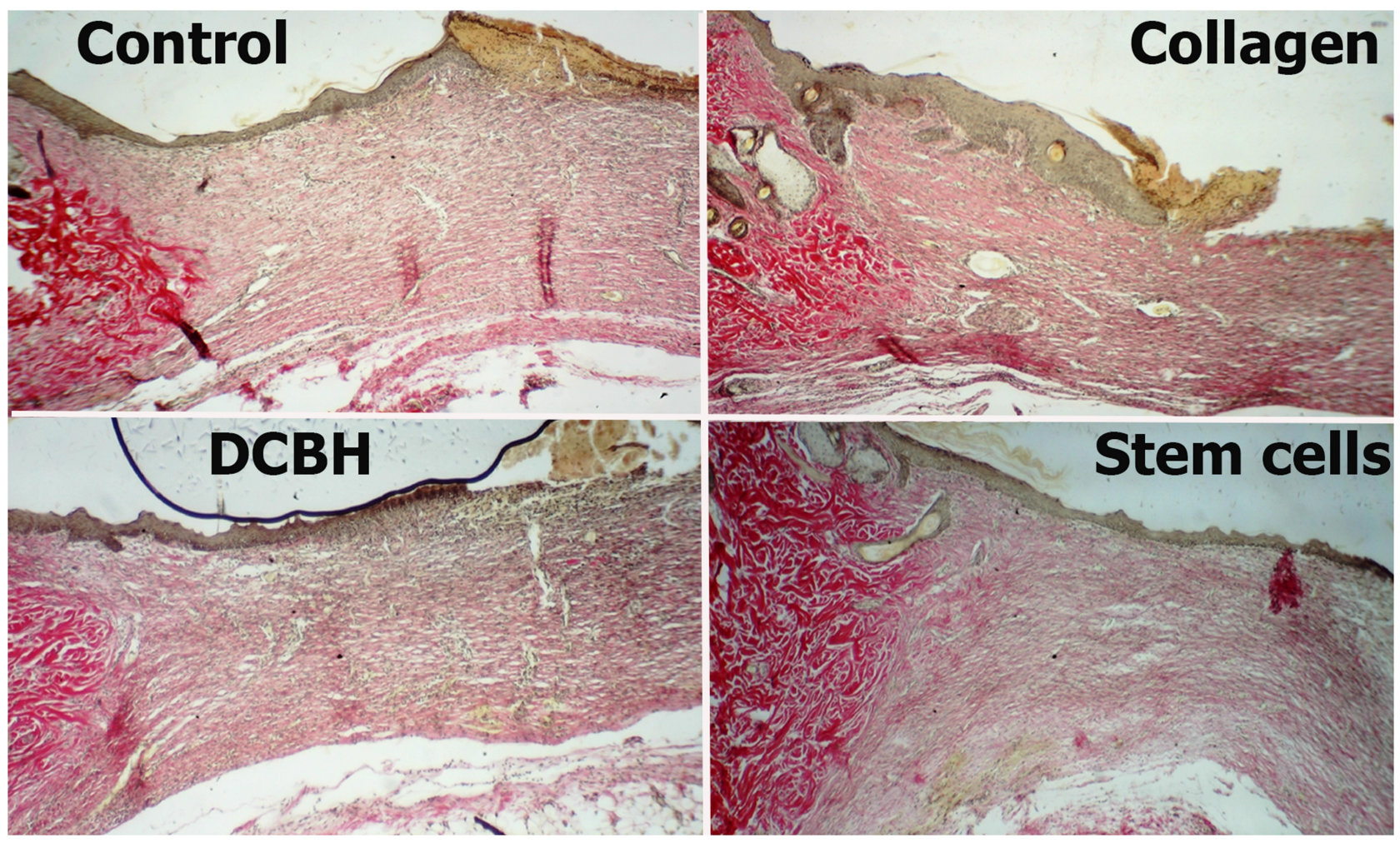

Figure 10 Tissues in the center and edges of the wounds in different rat groups on day 14. Van Gieson staining, magnification: $\times 40$. Abbreviation: $\mathrm{DCBH}$, deproteinized calf blood hemoderivative.

of mature collagen was detected in $14.3 \%$ of cases. The Control $(\mathrm{p}=0.014)$ and $\mathrm{SC}(\mathrm{p}=0.031)$ groups statistically significantly differed from the Collagen group in this indicator.

\section{Discussion}

The aim of the present study was to comparatively evaluate the effectiveness of the drugs used for wound healing regulation, which are injected into the wound center or its edges. A standardized skin damage model was used, which made it possible to compare the results of the treatment in animals classified in different pharmacological groups.

According to the wound area assessment, for 2 weeks, the largest wound area was observed in the Control group, which once again demonstrated the relative weakness of the evolutionarily developed wound healing mechanisms, and the effectiveness of modern pharmacological agents used for wound healing.

On day 3, the lowest wound area was reported in the SC group. The Collagen and DCBH groups wound sizes did not differ in size on day 3 , and did not differ versus other groups. On days 7 and 14, the wound size reduction was better in the Collagen group, and worse in the Control group. The SC and
DCBH wound size occupied an intermediate position and did not differ statistically from each other throughout the observation period. According to the wound sizes, the groups can be arranged in the following order: day 3 , Control $>$ Collagen $=\mathrm{DCBH}>\mathrm{SC}$; day 7 , Control $>\mathrm{SC} \geq \mathrm{DCBH}>$ Collagen; and day 14 , Control $>\mathrm{DCBH} \geq \mathrm{SC}>$ Collagen.

On day 3 , the fibroblast concentration in the wound tissues was the lowest in the SC group, followed by the Collagen group, and was highest in the Control group. However, the largest difference between the number of fibroblasts in the wound center and edges was noted precisely in the wounds of the SC and Collagen groups. In the wound center of the $\mathrm{SC}$ and Collagen groups the number of fibroblasts per unit area was $2-5 \times$ higher (in other groups: $1.1-1.5 \times$ higher). Furthermore, in the wound areas of the SC and Collagen groups, the absolute number of fibroblasts was the smallest; however, there was more newly formed collagen than in the other groups. Probably, collagenization and other protein-forming functions of fibroblasts at this stage of the wound process are associated with the presence of additional building material that accelerates the production of its own collagen and with the activity of fibroblasts rather than their quantity. 
It was found that the concentration of fibroblasts in the wound tissues is not reliably associated with the blood flow. The control wounds on day 3 had the highest cell infiltration; however, the level of microhemoperfusion did not change compared with that detected in the first 30-40 min after the wound modeling, ie, during the hemostasis stage. At the same time, the number of cells per unit area was statistically indistinguishable between the DCBH and Control groups. However, only in the DCBH group, an increase in the perfusion volume along the wound on day 3 was recorded; the observed increase confirmed the conclusions of one of our co-authors regarding the effect of DCBH on neoangiogenesis. ${ }^{31}$ Thus, the biological effect of serum substances in the blood of young animals, which act primarily under ischemic conditions, was confirmed. ${ }^{21}$

Thus, on day 3, the best wound healing observed in the SC group may be associated with the anti-inflammatory effect and stimulation of the protein-production processes of a wound's own fibroblasts. This confirmed the opinion of other researchers concerning the effectiveness of using allogeneic invisible mesenchymal SCs isolated from the human umbilical cord. ${ }^{28,30,32,33}$ The relatively successful regeneration of the DCBH wounds may be related to an increase in microvessel perfusion in the skin around the wound. However, the increase in perfusion volume recorded on day 3 in the DCBH group did not eventually have a significant effect on the wound healing. The shortterm effect of improving microcirculation in the DCBH group could occur due to the implementation of inflammation mechanisms. It is difficult to imagine the formed neoangiogenesis in the first day after injury. Inflammation is typical for this period. That is, an increase of microcirculation indicators could be associated not with neoangiogenesis, but with the expansion of existing capillaries, and the release of non-resident leukocyte cells into the tissue of wounds of the DCBH group. Moreover, later the effect of enhanced microcirculation disappeared. Our next works will be devoted to testing this hypothesis.

By the end of day 7, both of the aforementioned effects had disappeared. The best wound regeneration was noted in the Collagen group. The histological profile showed that, on day 7 , the best wound healing was detected in the wound edges of the SC and Collagen groups, where the formation of the wound surface epithelial coating began; the largest number of mature fibroblasts was recorded under the epidermis. As a result of the fibroblast prevalence in different wound sections, as well as the faster maturation of fibroblasts in the SC and Collagen groups, signs of more intensive collagen and epithelium formation and maturity were revealed in these groups in comparison with the DCBH and Control groups. In all wounds of the DCBH and Control groups, young collagen prevailed over mature collage in volume, which can be regarded as a sign of a relative slowdown in protein synthesis by fibroblasts.

Moreover, wound healing effectiveness is associated with microhemoperfusion. On day 7 in the SC and Collagen groups, an increased and a maximum perfusion volume, as well as the flow of blood elements, were recorded, which correlated with a decrease in the size of the wounds.

By the end of day 14, based on the wound area dynamics, it can be argued that wounds of the Collagen group and, to a somewhat lesser extent, those of the SC group healed more intensively. In terms of the wound surface epithelialization on day 14 , the best result was also recorded in the $\mathrm{SC}$ and Collagen groups. In the DCBH group, the level of epithelialization was similar to that observed in the Control group, and complete wound coverage with epidermis did not occur in any case. The concentration of fibroblasts on day 14 was highest in the center of the wound of the DCBH group, in the wound edges the concentration of fibroblasts was highest in the Collagen and DCBH groups. The highest and lowest degree of collagen maturity and organization on day 14 was recorded in the Collagen and Control groups, respectively. The blood flow in the wound edges increased significantly in all groups.

Wound surface epithelialization during the morphological assessment varied within the groups. Epithelium stratification was noted in the SC group and in the Collagen group, in which the skin derivatives were formed to the highest degree. According to the collagen organization and maturation, the groups may be arranged in the following ascending order: Control $>\mathrm{DCBH}>\mathrm{SC}>$ Collagen.

Thus, between days 7-14, wound healing in the Collagen group was the best in the indicators we use. This is due to the reduction in the time for a full cycle of collagen synthesis by fibroblasts in connection with the use of the introduced foreign collagen amino acids. This is also associated with the most rapid maturation of fibroblasts. The consistently high concentration of fibroblasts at the wound bottom and edge tissues in the Collagen group explains the acceleration of the wound healing process, which was initiated in the edges and at the bottom of 
wounds, reducing their depth. The positive effect of collagen that we have established experimentally confirms the clinical effectiveness of its use even in the treatment of chronic wounds. ${ }^{15-17}$ This allows us to conclude about the unity of the mechanisms of collagen influence on acute and chronic wound regeneration.

However, the process of physiological skin restoration is not limited to 14 days. It is possible that at a later date, after the completion of the remodeling process, we will receive different aesthetic and other characteristics of the formed scar. Indeed, after the closure of the wound, the issue of aesthetics and elasticity of the site of operation will be of greatest concern to the patient. This important question requires additional research.

A separate discussion is required for the treatment of chronic-infected wounds and the development of new highly effective injectable antibacterial agents, work on which is already underway. ${ }^{34-36}$ Success in this direction would make it possible to abandon the long-term use of antibiotics, and possibly to solve the problem of antibiotic resistance. In our study, wound infection did not occur, despite the aseptic conditions of wound modeling. This is due to acute wounds in highly immunocompromised animal models.

Thus, this study reveals some aspects of wound healing and confirms the need to develop new high-tech wound care products. Future directions of research in this area are attempts to create models of chronic wounds with and without infection, the study of pathophysiology with an analysis of the mechanisms and comparative efficacy of different drugs.

\section{Conclusion}

Despite the large number of options for the treatment of wounds, it is obvious that there cannot be a universal remedy. In this regard, the aim of treatment should be formulated as reasonably and clearly as possible: earliest possible closure of the wound; decrease in the possibility of ulcer recurrence; obtaining the maximum possible cosmetic effect, etc. This will allow clinicians to more accurately select the treatment scheme or a combined approach for the treatment of skin defects.

The results of this study showed that the best healing was noted on day 3 and days 7-14 in the SC and Collagen groups, respectively. Comprehensive monitoring of the wound condition is important both before and during treatment. A diagnostic method for this purpose is the assessment of microcirculation in wound edges. It was found that an increase in the volume of wound edge perfusion accelerates the regeneration rate, while the level of microcirculation is not associated with the number and activity of fibroblasts.

An instrumental study of the state of wound edges is an essential step towards the personalized treatment of somatic diseases accompanied by ulcers, as well as postoperative patient management in the presence of skin integrity disorders.

In conclusion, the effect of injectable drugs is realized through various biological and chemical mechanisms. In addition, the acceleration of regeneration is associated with the presence of a sufficient pool of mature and active fibroblasts in the wound, rather than their total number. Moreover, the use of SCs provided a rapid but shortlasting effect, whereas the use of Collagen resulted in a delayed (from day 7) but persistent and longer woundhealing effect. Taking into account the short lifespan of $\mathrm{SCs}$, it is advisable to re-introduce them into a wound to achieve a prolonged effect and the synthesis of biologically active substances that accelerate wound healing. Calf blood preparation (DCBH) can have a favorable effect in cases of tissue hypoxia. The effect of DCBH is the weakest on acute wound models; hence, further investigation is warranted. Instrumental control makes it possible to actualize the choice of the optimal drug or drug combination to improve and accelerate the wound healing process.

\section{The Main Findings of the Study}

1. The injectable drugs' effect is realized through various biological and chemical mechanisms.

2. The regeneration acceleration is associated with the presence of a sufficient pool of mature and active fibroblasts in the wound, and not with the total number of fibroblast cells.

3. The use of SC provided a quick but short effect, while the use of Collagen - a delayed (from the 7th day) but persistent and longer wound-healing effect. Taking into account the short period of SC lifetime, it is advisable to re-introduce them into a wound to create a prolonged effect and the synthesis of biologically active substances that accelerate wound healing. Calf blood preparation (DCBH) can have a good effect in cases of tissue hypoxia. On the acute wound models, the effect of DCBH is the weakest and requires further research. 
4. Instrumental control makes it possible to actualize the optimal drug or drug combination choice to improve and accelerate the wound healing process.

\section{Acknowledgments}

The author would like to thank everyone who participated in the study, including Artyushkova EB, Ivanov AV, Kryukov AA, Gladchenko MP, Khokhlov NV, Dudka VT, Furman YuV, Naimzada MDZ, Anikanov AV, Kaplin $\mathrm{AN}$, and Vasin VI.

\section{Funding}

This work did not receive specific sponsorship. The materials presented were obtained as a part of the Foundation for the Promotion of the Development of Small Forms of Enterprises in the Scientific and Technical Field grant (232GRNTIS5/35963), devoted to the development of a biomedical product for wound healing. The groups described in the work were comparison groups for products developed and funded by the grant.

\section{Disclosure}

The authors report no conflicts of interest in this work.

\section{References}

1. Beers EH. Palliative wound care: less is more. Surg Clin North Am. 2019;99(5):899-919. doi:10.1016/j.suc.2019.06.008

2. Haalboom M. Chronic wounds: innovations in diagnostics and therapeutics. Curr Med Chem. 2018;25(41):5772-5781. doi:10.2174/ 0929867324666170710120556

3. Han G, Ceilley R. Chronic wound healing: a review of current management and treatments. Adv Ther. 2017;34(3):599-610. doi:10.1007/ s12325-017-0478-y

4. Ennis WJ, Lee C, Gellada K, Corbiere TF, Koh TJ. Advanced technologies to improve wound healing: electrical stimulation, vibration therapy, and ultrasound-What is the evidence? Plast Reconstr Surg. 2016;138(3Suppl):94S-104S. doi:10.1097/PRS.0000000000002680

5. Zielins ER, Brett EA, Luan A, et al. Emerging drugs for the treatment of wound healing. Expert Opin Emerg Drugs. 2015;20(2):235-246. doi:10.1517/14728214.2015.1018176

6. Chen X, Zhang M, Wang X, et al. Peptide-modified chitosan hydrogels promote skin wound healing by enhancing wound angiogenesis and inhibiting inflammation. Am J Transl Res. 2017;9(5):2352-2362.

7. $\mathrm{Bi} \mathrm{H}$, Li $\mathrm{H}$, Zhang $\mathrm{C}$, et al. Stromal vascular fraction promotes migration of fibroblasts and angiogenesis through regulation of extracellular matrix in the skin wound healing process. Stem Cell Res Ther. 2019;10(1):302. doi:10.1186/s13287-019-1415-6

8. Velnar T, Gradisnik L. Tissue augmentation in wound healing: the role of endothelial and epithelial cells. Med Arch. 2018;72(6):444-448. doi:10.5455/medarh.2018.72.444-448

9. Miscianinov V, Martello A, Rose L, et al. MicroRNA-148b targets the TGF- $\beta$ pathway to regulate angiogenesis and endothelial-to-mesenchymal transition during skin wound healing. Mol Ther. 2018;26 (8):1996-2007. doi:10.1016/j.ymthe.2018.05.002
10. Lynch MD, Watt FM. Fibroblast heterogeneity: implications for human disease. J Clin Invest. 2018;128(1):26-35. doi:10.1172/ JCI93555

11. Thulabandu V, Chen D, Atit RP. Dermal fibroblast in cutaneous development and healing. Wiley Interdiscip Rev Dev Biol. 2018;7 (2). doi:10.1002/wdev.307

12. Bainbridge P. Wound healing and the role of fibroblasts. $J$ Wound Care. 2013;22(8):407-412. doi:10.12968/jowc.2013.22.8.407

13. Sun M, Wang P, Okubo T, et al. Possible contribution of fibrocytes to increased type I collagen synthesis during the early stage of dermal wound repair in human skin. $J$ Invest Dermatol. 2018;138(1):240242. doi:10.1016/j.jid.2017.08.020

14. Stunova A, Vistejnova L. Dermal fibroblasts-A heterogeneous population with regulatory function in wound healing. Cytokine Growth Factor Rev. 2018;39:137-150. doi:10.1016/j. cytogfr.2018.01.003

15. Stupin VA, Silina EV, Goryunov SV, et al. Assessment of changes in the lesions sizes and the incidence of complete epithelialization during the treatment of diabetic foot syndrome over a period of 4 weeks (multicenter study). Khirurgiia (Mosk). 2017;3:55-60. doi:10.17116/hirurgia2017355-60

16. Dibirov MD, Gadzhimuradov RU, Koreyba KA. Analysis of the clinical application of epidermal growth factor ("Heberprot-P") and bioplastic material ("Collost") in treatment of skin and soft tissues defects in patients with diabetic foot syndrome. Khirurgiia (Mosk). 2016;3:59-63. doi:10.17116/hirurgia2016359-63

17. Stupin VA, Gabitov RB, Sinelnikova TG, Silina EV. Biological mechanisms of chronic wound and diabetic foot healing: the role of collagen. Serbian J Exp Clin Res. 2018;19(4):373-382. doi:10.2478/ sjecr-2018-0077

18. Manturova NE, Stenko AG, Petinati Y, Chaikovskaya EA, Bolgarina AA. Injectable collagen in correction of age-related skin changes: experimental and clinical parallels. Bull Russ State Medical Univ. 2019;8(1):71-77. doi:10.24075/BRSMU.2019.010

19. Budkevich LI, Mirzoyan GV, Gabitov RB, Shmyrin AA, Glutkin AV. Collost bioplastic collagen material for the treatment of burns. Sovrem Tekhnologii Med. 2020;12(1):92-96. doi:10.17691/ stm2020.12.1.12

20. Brock J, Golding D, Smith PM, Nokes L, Kwan A, Lee PYF. Update on the role of actovegin in musculoskeletal medicine: a review of the past 10 years. Clin J Sport Med. 2020;30(1):83-90. doi:10.1097/ JSM.0000000000000566

21. Firan FC, Romila A, Onose G. Current synthesis and systematic review of main effects of calf blood deproteinized medicine (Actovegin $^{\circledR}$ ) in ischemic stroke. Int J Mol Sci. 2020;21(9):3181. doi:10.3390/ijms21093181

22. Belikan P, Nauth L, Färber LC, et al. Intramuscular injection of combined calf blood compound (CFC) and homeopathic drug $\operatorname{Tr} 14$ accelerates muscle regeneration in vivo. Int $J$ Mol Sci. 2020;21 (6):2112. doi:10.3390/ijms 21062112

23. Lee DE, Ayoub N, Agrawal DK. Mesenchymal stem cells and cutaneous wound healing: novel methods to increase cell delivery and therapeutic efficacy. Stem Cell Res Ther. 2016;7:37. doi:10.1186/ s13287-016-0303-6

24. Kanji S, Das H. Advances of stem cell therapeutics in cutaneous wound healing and regeneration. Mediators Inflamm. 2017;2017:5217967. doi:10.1155/2017/5217967

25. Maranda EL, Rodriguez-Menocal L, Badiavas EV. Role of mesenchymal stem cells in dermal repair in burns and diabetic wounds. Curr Stem Cell Res Ther. 2017;12(1):61-70. doi:10.2174/ $1574888 \times 11666160714115926$

26. Guo J, Hu H, Gorecka J, et al. Adipose-derived mesenchymal stem cells accelerate diabetic wound healing in a similar fashion as bone marrow-derived cells. Am J Physiol Cell Physiol. 2018;315(6):C885C896. doi:10.1152/ajpcell.00120.2018 
27. Huang YZ, Gou M, Da LC, Zhang WQ, Xie HQ. Mesenchymal stem cells for chronic wound healing: current status of preclinical and clinical studies. Tissue Eng Part B Rev. 2020. doi:10.1089/ten. TEB.2019.0351

28. Suzdaltseva Y, Zhidkih S, Kiselev SL, Stupin V. Locally delivered umbilical cord mesenchymal stromal cells reduce chronic inflammation in long-term nonhealing wounds: a randomized study. Stem Cells Int. 2020;2020:5308609. doi:10.1155/2020/5308609

29. Yarygin KN, Suzdal'tseva YG, Burunova VV, et al. Comparative study of adult human skin fibroblasts and umbilical fibroblast-like cells. Bull Exp Biol Med. 2006;141(1):161-166. doi:10.1007/s10517006-0117-0

30. Manturova NE, Smirnova GO, Silina EV, Suzdaltseva YG, Stupin VA. The effectiveness of mesotherapy with progenitor cells of involutional changes in facial skin in patients of different ages. Ann Trop Med Public Health. 2018;9:S614.

31. Orlova AY, Sukovatykh BS, Artyushkova EB, Gordov MY. Vliyanie aktovegina i solkoserila na mikrocirkulyaciyu pri kriticheskoj ishemii nizhnih konechnostej $\mathrm{v}$ eksperimente. [The influence of actovegin and solcoseryl on microcirculation in critical ischemia of the lower extremities in the experiment]. Angiol Vasc Surg. 2017;23(1):29-35.

32. Fang S, Xu C, Zhang Y, et al. Umbilical cord-derived mesenchymal stem cell-derived exosomal microRNAs suppress myofibroblast differentiation by inhibiting the transforming growth factor- $\beta / \mathrm{SMAD} 2$ pathway during wound healing. Stem Cells Transl Med. 2016;5 (10):1425-1439. doi:10.5966/sctm.2015-0367
33. Han Y, Sun T, Han Y, et al. Human umbilical cord mesenchymal stem cells implantation accelerates cutaneous wound healing in diabetic rats via the Wnt signaling pathway. Eur J Med Res. 2019;24(1):10. doi:10.1186/s40001-019-0366-9

34. Liang $\mathrm{Y}$, Zhao $\mathrm{X}, \mathrm{Hu} \mathrm{T}$, et al. Adhesive hemostatic conducting injectable composite hydrogels with sustained drug release and photothermal antibacterial activity to promote full-thickness skin regeneration during wound healing. Small. 2019;15(12):e1900046. doi:10.1002/smll.201900046

35. Qu J, Zhao X, Liang Y, Zhang T, Ma PX, Guo B. Antibacterial adhesive injectable hydrogels with rapid self-healing, extensibility and compressibility as wound dressing for joints skin wound healing. Biomaterials. 2018;183:185-199. doi:10.1016/j. biomaterials.2018.08.044

36. Liang Y, Chen B, Li M, He J, Yin Z, Guo B. Injectable antimicrobial conductive hydrogels for wound disinfection and infectious wound healing. Biomacromolecules. 2020;21(5):1841-1852. doi:10.1021/ acs.biomac.9b01732
Drug Design, Development and Therapy

\section{Publish your work in this journal}

Drug Design, Development and Therapy is an international, peerreviewed open-access journal that spans the spectrum of drug design and development through to clinical applications. Clinical outcomes, patient safety, and programs for the development and effective, safe, and sustained use of medicines are a feature of the journal, which has also

\section{Dovepress}

been accepted for indexing on PubMed Central. The manuscript management system is completely online and includes a very quick and fair peer-review system, which is all easy to use. Visit http://www. dovepress.com/testimonials.php to read real quotes from published authors. 